\title{
ENSAYO METODOLÓGICO PARA LA VALORACIÓN ESTÉTICA DEL PAISAJE. APLICACIÓN EN MUNTANYES D’ORDAL (BARCELONA)*
}

\author{
David Serrano Giné \\ Servei de Gestió i Evolució del Paisatge \\ Facultat de Geografía i Història (Barcelona) \\ dvdsig@gmail.com
}

\begin{abstract}
Resumen: Uno de los aspectos más en boga en los estudios de paisaje se refiere a las técnicas y métodos de valoración estética. Por norma general, estos ejercicios acostumbran a tildarse de subjetivos y poco sistemáticos, hecho que suele reducir su interés y aplicabilidad. A partir de una metodología indirecta basada en la agregación ponderada de elementos, se realiza una valoración estética del paisaje de Muntanyes d'Ordal, un espacio periurbano cercano a la ciudad de Barcelona.
\end{abstract}

Palabras clave: paisaje, valoración estética, Muntanyes d'Ordal.

\begin{abstract}
Among landscape studies, one of the aspects of most interest refers to techniques and methods to consider values of aesthetic type. By general rule, these exercises are used to be considered subjective and little systematic, fact that can reduce its interest and applicability. From an indirect methodology based on the weighed aggregation of elements, an aesthetic valuation of landscape is developed at Muntanyes d'Ordal, a periurban area near the city of Barcelona.
\end{abstract}

Keywords: landscape, aesthetic valuation, Muntanyes d'Ordal.

\section{Introducción}

Como es sabido, el término paisaje presenta una amplia diversidad de significados y de planteamientos de estudio (González Bernáldez, 1981; Bolòs, 1992).

* Recibido: 7-7-08. Aceptado: 9-1-09. 
Actualmente dicha polisemia converge en el llamado renovado interés por el paisaje. En esta revalorización ha contribuido ostensiblemente la Convención europea del paisaje (CEP), firmada en Florencia en octubre de 2000. La CEP entiende por paisaje "cualquier parte del territorio, tal como es percibida por las poblaciones, cuyo carácter resulta de la acción de factores naturales y/o humanos y de sus interrelaciones". En su preámbulo asegura que “...el paisaje es en todas partes un elemento importante de la calidad de vida de las poblaciones, tanto en los medios urbanos como rurales, en los territorios degradados como en los de gran calidad, en los espacios singulares como en los cotidianos", para más tarde afirmar que "...el paisaje constituye un elemento esencial del bienestar individual y social, y que su protección, su gestión y su ordenación implican derechos y responsabilidades para cada persona".

Adoptando la CEP como referencia, son numerosos los autores que han destacado la popularización y revalorización del paisaje y que han relacionado el resurgimiento de temáticas de paisaje con distintas inquietudes de tipo social y territorial. De esta manera, Zoido (2002) indica la importancia del paisaje en las políticas públicas y, particularmente, en la ordenación del territorio. Otros autores, como Cañellas et al. (2006), señalan su creciente importancia política e institucional, así como las consecuencias que de ello se derivan en el ámbito de la Administración pública y la participación ciudadana.

Por otra parte, Tarroja (2006) apunta que la popularidad y la importancia social del paisaje pueden explicarse por las transformaciones territoriales y la falta de tradición en la gestión de espacios abiertos no protegidos, las recientes movilizaciones colectivas sobre las transformaciones del entorno y las iniciativas noveles de incorporación del paisaje en la ordenación y gestión del territorio. Es por todo ello que, siguiendo la CEP, no son pocas las voces que señalan un rol significativo del paisaje como indicador de la calidad de un territorio y, también, de la calidad de vida de las personas; en esta línea, reconocidos autores aprecian dicha dimensión, que permite considerar el paisaje como un componente esencial del espacio vivido (Mata et al., 2001).

Como ya se ha avanzado, existen distintas vías para acercarse al paisaje desde un punto de vista académico. La distinción de Zoido y Posocco (1998) apuesta por diferenciar tres grandes perspectivas: una primera, centrada en su dimensión territorial; una segunda, preocupada por la intervención; y una tercera, ocupada en los aspectos subjetivos percibidos y asimilados por el individuo. Este último planteamiento ha dado lugar a numerosos trabajos, tanto de carácter teórico como aplicado (Cendrero, 1986; González Bernáldez, 1993; Gómez y Fernández, 1999; Scott, 2002; Martínez de la Vega et al., 2003; Arriaza et al., 2004; Ode et al., 2008), que aumentan su interés si se considera el peso que ostenta el paisaje en la calidad de vida y el bienestar de personas y sociedades.

Se conoce un buen número de técnicas para evaluar las características visuales y el valor estético de un paisaje. Por norma general se acostumbra a diferenciar entre métodos directos y métodos indirectos, según la importancia que se otorgue al obser- 
vador en la calificación, primero, y la valoración, después, del paisaje. Mallarach (1999) advierte que los métodos directos se basan en contrastar las preferencias de una población muestral con el objetivo de llegar a un consenso, mientras que los métodos indirectos se centran en determinar la presencia e intensidad de aquellos elementos del paisaje a los que se les atribuye valor estético. Otras clasificaciones más detalladas desmenuzan esta división en cinco perspectivas de trabajo: métodos de análisis de componentes, de predicción de preferencias, de contemplación de la totalidad, de valoración directa y análisis posterior de las componentes y de valoración económica (García y Cañas, 2001). Aparte, conviene considerar los diferentes métodos ocupados en captar actitudes y pareceres de orden social o individual (Nogué, 1986), o que focalizan en la importancia del paisaje en las políticas públicas, particularmente cuando afectan a poblaciones locales (Vaccaro y Norman, 2008).

En cualquier caso, el mayor número de críticas a este tipo de trabajos se centra en el elevado grado de subjetividad que se les asocia, ya sea en la representatividad de la población muestral (en el caso de los métodos directos), ya en la oportunidad y valoración de los elementos de referencia escogidos (en el caso de los métodos indirectos). Es por ello que no existen metodologías universales y excluyentes, que la valoración de un paisaje determinado siempre se realiza por comparación con otro de referencia y que, dada la subjetividad inherente a todos los procedimientos, la sistemática es el único requisito necesario para cualquier método (Zube, 1984).

\section{Objetivos}

El objeto de este ejercicio es realizar una valoración estética del paisaje de Muntanyes d'Ordal, partiendo de la delimitación detallada de unidades de paisaje y utilizando una metodología de tipo indirecto basada en la agregación ponderada de elementos.

El método utilizado se basa en las experiencias desarrolladas en las provincias del País Valenciano (Cendrero, 1986; INGEMISA, 1987, 1991) y en el trabajo coordinado por Juan Sánchez (1995) en Gran Canaria. También nos hemos orientado a partir de otros estudios, centrados en analizar y establecer las preferencias de paisaje por parte de la población (Rochefort, 1974; González Bernáldez, 1981; Purcell, 1992; Gómez y Fernández, 1999; Arriaza et al., 2004; De la Fuente et al., 2006) donde, de manera global, se converge en valorar positivamente los espacios con vegetación fragosa, las formaciones arbóreas bien desarrolladas, el relieve accidentado, la existencia de láminas de agua y los mosaicos paisajísticos, frente a lugares secos, privados de cubierta vegetal, topografías suaves y configuraciones homogéneas.

En concreto, hemos aplicado un procedimiento de agregación simple ponderada sin valores negativos, según la terminología de Mallarach (1999). La intención del método es disgregar los elementos que estructuran con mayor peso los valores estéticos del paisaje, ponderarlos según unos criterios de referencia y relacionarlos a tra- 
vés de una estadística sencilla. Se ha realizado una doble calificación, según la calidad y la fragilidad de cada elemento. La calidad se ha establecido a partir de los elementos intrínsecos de un paisaje y en función a unos parámetros de referencia. Por el contrario, por fragilidad se ha entendido el riesgo de deterioro de la calidad, generalmente como consecuencia de la implantación de actividades humanas; éste es un concepto similar al de vulnerabilidad visual y opuesto al de capacidad de absorción visual (AAVV, 2004).

\section{3. Área de estudio}

El estudio se desarrolla en el sector norte y centro de Muntanyes d'Ordal, en un espacio ligeramente superior a los $150 \mathrm{~km}^{2}$ repartido en trece municipios, todos pertenecientes a la región metropolitana de Barcelona (Figura 1).

El rasgo principal del lugar es la sucesión de valles y sierras de poca envergadura (Montau, 658,1m; Puig d'Agulles, 653,1m), pero nítidamente diferenciados de los lla-

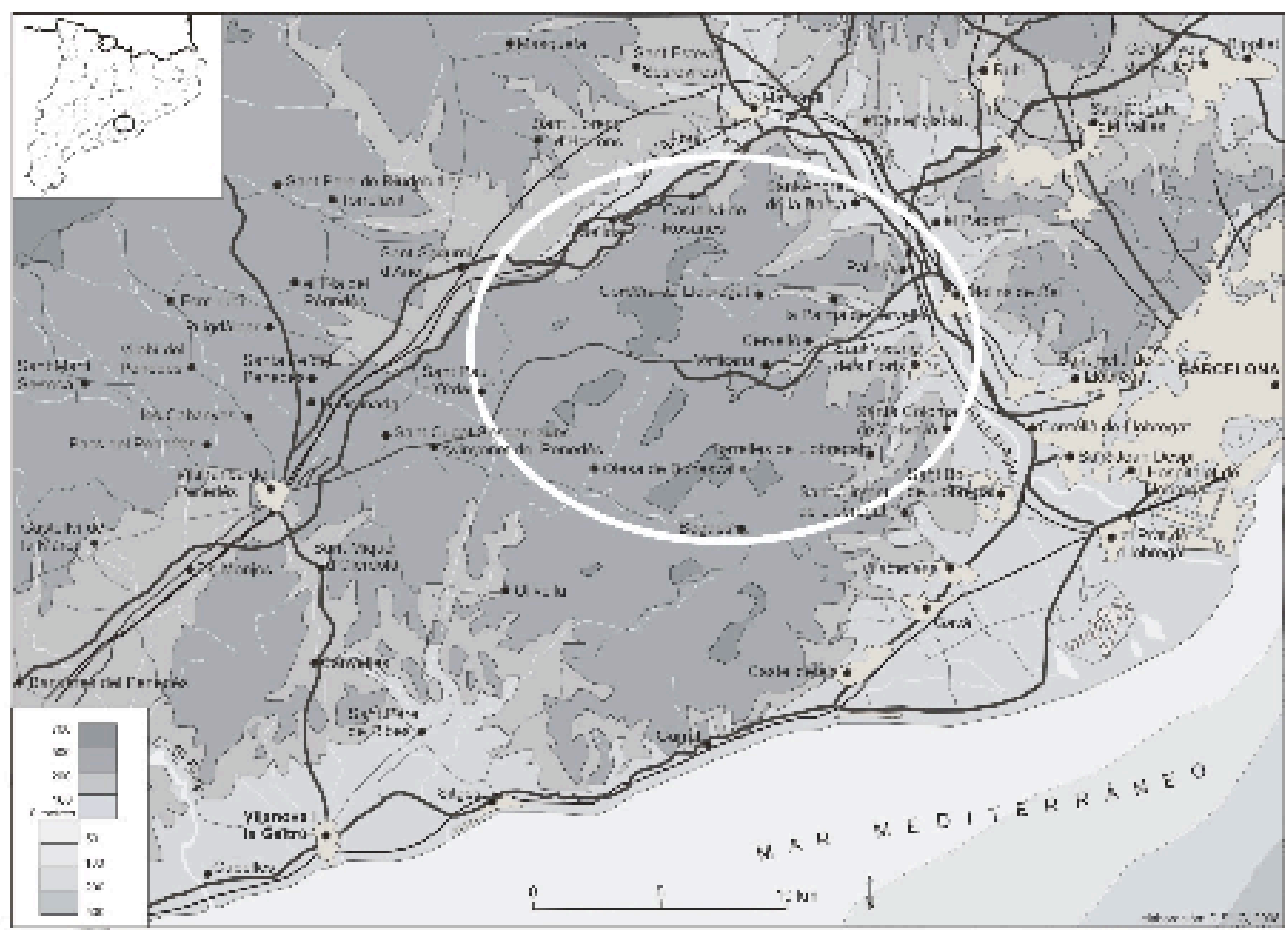

Figura 1. Localización del área de estudio. Fuente: elaboración propia. 
nos circundantes, como el delta y el tramo bajo del Llobregat o la depresión del Penedés. La cubierta principal se constituye mayormente por formaciones boscosas de Pinus halepensis y Quercus ilex, en combinación con otras especies de carácter mediterráneo; en la parte occidental de las montañas abundan las formaciones arbustivas, particularmente máquias de Quercus coccifera (Figura 2).

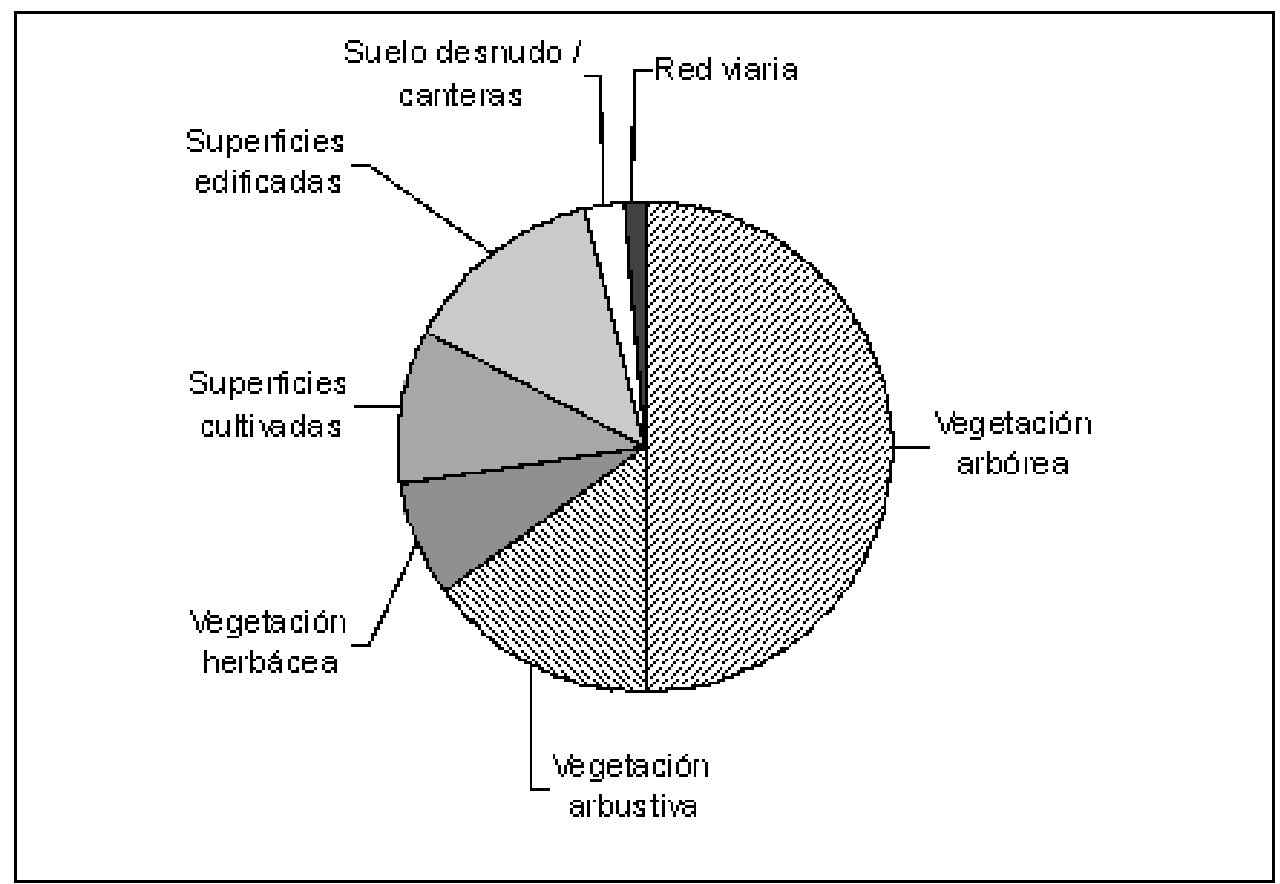

Figura 2. Ocupación del suelo. Fuente: elaboración propia a partir de fotointerpretación.

Hasta la década de 1950 la dedicación económica principal se basaba en la agricultura de secano, siendo la vid el cultivo más extendido. Los distintos cambios económicos y sociales ocurridos en España a partir de mediados de siglo XX, condujeron a la aparición de un rosario de urbanizaciones residenciales de baja densidad, habitualmente nacidas de manera irregular y, más tarde, legalizadas (Paül y Serrano, 2005). Consecuencia de ello es la existencia de más de sesenta urbanizaciones, ideadas bajo el modelo de ciudad jardín, durante años concretizadas por la autoconstrucción y hasta fechas recientes faltas de los servicios básicos de cualquier actuación urbanística. Los espacios edificados, en conjunto, significan un 17,15\% de la superficie de las sierras; un $77,64 \%$ de esta cifra se corresponde con urbanizaciones de carácter extensivo. Precisamente esta ocupación del suelo, a pesar de ser minoritaria, es la que marca con más fuerza el carácter y la fisonomía de Muntanyes d’Ordal. 
De un tiempo a esta parte, distintos movimientos asociativos en defensa del territorio han reclamado mayor atención para los valores naturales y culturales de este espacio (Serrano y Piquer, 2006). Parte de estas aspiraciones han sido reconocidas por la Administración, incluyendo algunos sectores del lugar en el Pla d'Espais d'Interès Natural de la Generalitat de Cataluña.

\section{Metodología}

La metodología seguida se divide en tres partes: la delimitación de unidades de paisaje, la caracterización de cada unidad y la ponderación de elementos.

\subsection{Delimitación de unidades de paisaje}

La base del estudio se encuentra en una cuidadosa delimitación de unidades de paisaje. Éstas se han establecido según criterios de homogeneidad, dinámica y funcionalidad, tienen validez cartográfica a escala 1:10 000 y han sido caracterizadas según la metodología expuesta por Bolòs (1992), que se basa en el dominio o, si procede, predominio de elementos y energías. La delimitación de las unidades se ha realizado mediante un exhaustivo trabajo de campo, desarrollado entre los meses de enero y abril de 2007.

\subsection{Caracterización de cada unidad}

De manera simultánea a la delimitación de unidades, se ha realizado una recogida sistemática de información con interés para la valoración estética del paisaje. Para ello ha sido necesario el uso de una ficha de campo estandarizada, que permitiera recoger siempre el mismo tipo de información y bajo los mismos criterios rectores.

\subsection{Ponderación de elementos}

Finalmente, se ha desarrollado la ponderación de elementos con interés en la valoración estética del paisaje. La escala de valores utilizada en la ponderación contempla el valor 1 para los elementos con menor interés y el valor 5 para los elementos con mayor interés; de esta manera, el valor 1 es equivalente a calidad muy baja, 2 a baja, 3 a media, 4 a alta y 5 a muy alta. Conviene aclarar que la asignación de pesos se ha realizado a partir de lo sugerido por la bibliografía específica, partiendo del conocimiento previo del área de estudio, comprobando la validez de las ponderaciones mediante un procedimiento de ensayo y error y corrigiendo las desviaciones aparecidas. Los elementos considerados han sido el relieve, los puntos de agua, la 
vegetación, las superficies cultivadas, los elementos patrimoniales y las superficies construidas.

\subsubsection{Relieve}

El único elemento del relieve que se ha considerado han sido los acantilados. Se ha distinguido entre acantilados en calizas (de coloración blanquecina) y en areniscas (de coloración rojiza), ya que la inclusión de otros elementos relacionados con la pendiente, alteraba los resultados finales (Tabla 1).

Tabla 1. Ponderación del relieve.

\begin{tabular}{|lcc|}
\hline \multicolumn{1}{|c}{ Elemento } & Calidad & Fragilidad \\
\hline Acantilados en calizas & 2 & 3 \\
Acantilados en areniscas & 3 & 4 \\
\hline
\end{tabular}

\subsubsection{Puntos de agua}

La presencia de puntos de agua se ha contemplado desde una doble perspectiva, según si el agua se encontraba estancada o en movimiento (Tabla2)

Tabla 2. Ponderación de los puntos de agua.

\begin{tabular}{|lcc|}
\hline \multicolumn{1}{|c}{ Elemento } & Calidad & Fragilidad \\
\hline Agua estancada & 4 & 2 \\
Agua corriente & 3 & 2 \\
\hline
\end{tabular}

\subsubsection{Vegetación}

La vegetación se ha considerado desde tres perspectivas distintas: la riqueza de estratos vegetales (Tabla 3), el tipo de formación vegetal (Tabla 4) y la presencia de especies caducifolias en número superior al 10\% de la unidad (Tabla 5).

Tabla 3. Ponderación de la vegetación (I).

\begin{tabular}{|lcc|}
\hline \multicolumn{1}{|c|}{ Elemento } & Calidad & Fragilidad \\
\hline Herbáceo & 1 & 5 \\
Subarbustivo & 2 & 4 \\
Arbustivo & 3 & 3 \\
Arborescente & 4 & 2 \\
Arbóreo & 5 & 1 \\
\hline
\end{tabular}


Tabla 4. Ponderación de la vegetación (II)

\begin{tabular}{|c|c|c|c|c|c|}
\hline Elemento & Calidad & Fragilidad & Elemento & Calidad & Fragilidad \\
\hline $\begin{array}{l}\text { Pinar de pino } \\
\text { blanco con } \\
\text { Rhamnus } \\
\text { alaternus }\end{array}$ & 5 & 2 & $\begin{array}{l}\text { Encinar con } \\
\text { Quercus } \\
\text { cerrioides }\end{array}$ & 5 & 1 \\
\hline $\begin{array}{l}\text { Pinar de pino } \\
\text { blanco con } \\
\text { Pistacea } \\
\text { lentiscus }\end{array}$ & 5 & 3 & Alcornocal & 5 & 1 \\
\hline $\begin{array}{l}\text { Pinar de pino } \\
\text { blanco con } \\
\text { Juniperus } \\
\text { oxycedrus }\end{array}$ & & & $\begin{array}{l}\text { Robledal de } \\
\text { Quercus } \\
\text { cerrioides }\end{array}$ & & \\
\hline $\begin{array}{l}\text { Pinar de pino } \\
\text { blanco con } \\
\text { Quercus } \\
\text { coccifera }\end{array}$ & 5 & 1 & $\begin{array}{l}\text { Vegetación } \\
\text { de ribera }\end{array}$ & 5 & 2 \\
\hline $\begin{array}{l}\text { Pinar de pino } \\
\text { blanco con } \\
\text { Ampelodesma } \\
\text { mauritanica }\end{array}$ & 5 & 1 & Cañar & 1 & 5 \\
\hline $\begin{array}{l}\text { Pinar de pino } \\
\text { blanco con } \\
\text { Erica arborea }\end{array}$ & 5 & 1 & $\begin{array}{l}\text { Matorral de } \\
\text { brezos con } \\
\text { pino blanco }\end{array}$ & 3 & 4 \\
\hline $\begin{array}{l}\text { Pinar de pino } \\
\text { piñonero }\end{array}$ & 5 & 1 & $\begin{array}{l}\text { Matorral de } \\
\text { brezos con } \\
\text { pino piñonero }\end{array}$ & 3 & 4 \\
\hline Encinar & 5 & 1 & Máquia & 4 & 3 \\
\hline $\begin{array}{l}\text { Pinar de pino } \\
\text { blanco con } \\
\text { encina }\end{array}$ & 5 & 1 & $\begin{array}{l}\text { Máquia } \\
\text { Spartium } \\
\text { junceum }\end{array}$ & 2 & 5 \\
\hline $\begin{array}{l}\text { Pinar de pino } \\
\text { piñonero con } \\
\text { encinar }\end{array}$ & 5 & 1 & Herbazal & 1 & 5 \\
\hline
\end{tabular}

Tabla 5. Ponderación de la vegetación (III).

\begin{tabular}{|lcc|}
\hline Elemento & Calidad & Fragilidad \\
\hline Caducifolios & 4 & 2 \\
\hline
\end{tabular}




\subsubsection{Superficies cultivadas}

Las superficies cultivadas se han desagregado en tres categorías: campos recientemente abandonados, agricultura de regadío y agricultura de secano (Tabla 6).

Tabla 6. Ponderación de las superficies cultivadas.

\begin{tabular}{|lcc|}
\hline \multicolumn{1}{|c}{ Elemento } & Calidad & Fragilidad \\
\hline Campos recientemente & 1 & 5 \\
abandonados & 3 & 1 \\
Campos de regadío & 5 & 2 \\
Campos de secano & \\
\hline
\end{tabular}

\subsubsection{Elementos patrimoniales}

Los elementos patrimoniales se han dividido en seis categorías: masías (M), iglesias o ermitas (I), hornos de cal o de vidrio (H), cabañas de piedra seca (C), paredes y muros de piedra seca (P) y otros (O) (Tabla 7). Con todo, a menudo en una misma unidad coincide más de un elemento, hecho que ha obligado a sistematizar la diversidad de casuísticas (Tabla 8) para poder establecer un criterio común (Tabla 9). Aparte, también se ha tenido en cuenta el estado de conservación de cada elemento, a partir de cuatro categorías de referencia: ruinas, malo, aceptable y bueno (Tabla 10).

Tabla 7. Ponderación de elementos patrimoniales (I).

\begin{tabular}{|c|c|c|}
\hline Elemento & Calidad & Fragilidad \\
\hline Masía & 5 & 2 \\
\hline Iglesia & 5 & 2 \\
\hline Horno/cabaña & 2 & 1 \\
\hline Paredes & 1 & 1 \\
\hline Otros & 3 & 3 \\
\hline
\end{tabular}

Tabla 8. Ponderación de elementos patrimoniales (II).

\begin{tabular}{|lc|}
\hline \multicolumn{1}{|c|}{ Elemento } & Valor \\
\hline M+I & 4 \\
M ó I + H ó C ó P & 3 \\
M ó I + H y C y P & 3 \\
M ó I + O & 4 \\
H ó C ó P + F ó Có P & 2 \\
H y C y P ó H ó Có P + & 2 \\
H y C y P ó H ó Có P & \\
H y C y P & 1 \\
O + H ó Có P & 3 \\
\hline
\end{tabular}


Tabla 9. Ponderación de elementos patrimoniales (III).

\begin{tabular}{|lccc|}
\hline & Valor del elemento & Calidad & Fragilidad \\
\hline 1 & 2 & 2 \\
2 & 3 & 2 \\
3 & 4 & 2 \\
4 & 5 & 1 \\
\hline
\end{tabular}

Tabla 10. Ponderación de elementos patrimoniales (IV).

\begin{tabular}{|c|c|c|}
\hline Elemento & Calidad & Fragilidad \\
\hline Ruinas & 1 & 5 \\
\hline Malo & 2 & 4 \\
\hline Aceptable & 3 & 2 \\
\hline Bueno & 5 & 1 \\
\hline
\end{tabular}

\subsubsection{Superficies construidas}

Las superficies construidas hacen referencia a las unidades caracterizadas por la edificación y las actividades antrópicas sin carácter patrimonial o de bien común. Se han considerado cuatro categorías: superficies urbanas compactas, superficies urbanas laxas próximas a superficies urbanas compactas, superficies urbanas laxas aisladas, superficies industriales, logísticas o de servicios, vertederos, canteras y superficies cimentadas (Tabla 11).

Tabla 11. Ponderación de las superficies construidas.

\begin{tabular}{|lcc|}
\hline \multicolumn{1}{|c}{ Elemento } & Calidad & Fragilidad \\
\hline Urbano compacto & 3 & 4 \\
Urbano laxo cerca de & 2 & 3 \\
compacto & & \\
Urbano laxo aislado & 1 & 2 \\
Industria, servicios, & 1 & 5 \\
vertederos, superficies & & \\
cimentadas & & \\
\hline
\end{tabular}

\subsubsection{Ponderación final}

Resulta evidente que los distintos elementos en que se ha simplificado el valor estético del paisaje no tienen un mismo peso, ni participan de la misma manera en la percepción del conjunto. Es por ello que nos ha parecido razonable aplicar un procedi- 
miento de agregación ponderada para cada elemento, asignándole un peso relativo que refleja su contribución al valor de la unidad (Tabla 12). A continuación se han

Tabla 12. Ponderación final.

\begin{tabular}{|lclc|}
\hline \multicolumn{1}{|c}{ Elemento } & Peso & \multicolumn{1}{c|}{ Elemento } & Peso \\
\hline Relieve & 5 & $\begin{array}{l}\text { Superficies } \\
\text { cultivadas }\end{array}$ & 3 \\
\hline Puntos de agua & 1 & $\begin{array}{l}\text { Elementos } \\
\text { patrimoniales }\end{array}$ & 4 \\
\hline $\begin{array}{l}\text { Estratos de } \\
\text { vegetación }\end{array}$ & 2 & $\begin{array}{l}\text { Estado de } \\
\text { conservación }\end{array}$ & 3 \\
\hline $\begin{array}{l}\text { Tipo de vegetación } \\
\text { Existencia de }\end{array}$ & 5 & $\begin{array}{l}\text { Superficies } \\
\text { construidas }\end{array}$ & 1 \\
\hline $\begin{array}{l}\text { Eaducifolios } \\
\text { caduct }\end{array}$ & 2 & & \\
\hline
\end{tabular}

calculado el índice de calidad (Iq) y de fragilidad (If) para cada clase, según la expresión que sigue:

$$
\mathrm{Iq}=\left(\sum \mathrm{Pe}\right)^{*} \mathrm{Vt} \quad \mathrm{If}=\left(\sum \mathrm{Pe}\right)^{*} \mathrm{Vt}
$$

Donde $I q$ es el índice de calidad y If el índice de fragilidad; Pe es el coeficiente otorgado a cada elemento $e$ y $V t$ es el valor del tipo de elemento $e$.

Finalmente, la calidad y la fragilidad se han sintetizado en una expresión conjunta, que combina ambos índices y da lugar al valor estético (Ve) del paisaje.

$$
\frac{\mathrm{Ve}=2 \mathrm{Iq}+\mathrm{If}}{3}
$$

Según esta expresión, la calidad del paisaje es un factor de mayor peso que la fragilidad. Esto es debido a que, en el área de estudio, las unidades no se valoran principalmente por el riesgo de degradación potencial, sino por el valor intrínseco que poseen.

\section{Resultados}

Según el método seguido, las áreas de valor estético muy bajo se encuentran en el valle del Llobregat, parte de la orilla del Anoia, el sector central del área de estudio (valle de Corbera y de la Palma) y en una franja que se alarga desde la unión de la rambla de Rafamans con el Llobregat hasta las canteras del llano de Ardenya, ocu- 
pando un 19,3\% de las unidades tratadas (Figura 3). Los paisajes de calidad baja significan un 18,9\% del total y tienen una distribución bastante irregular por todo el sector, si bien son más abundantes en el tercio norte; se corresponden con urbanizaciones que acogen algún elemento patrimonial, con zonas boscosas degradadas y maquias.

Hacia poniente y, en general, por todas las sierras se cuentan unidades con un valor estético medio (32,5\% de las unidades); estadísticamente se justifican en campos de cultivo de secano, principalmente viñas, pero también zonas boscosas poco espesas y máquias. Las unidades de calidad alta (20\% del total) se concentran, sobre todo, en el corazón de las montañas y se corresponden con formaciones boscosas bien desarrolladas y pluriestratificadas, a menudo con presencia de caducifolios, acantilados calizos y presencia de elementos patrimoniales. Un 9,2\% de las unidades tienen una valoración muy alta, acostumbran a distribuirse dispersas y aparecen particularmente agrupadas hacia el norte. También hacen referencia a bosques maduros y espesos, con presencia de caducifolios y frecuentemente con elementos patrimoniales, también atienden a acantilados triásicos y a maquias y "brollas" bien desarrolladas y con afloramientos rocosos.

La bondad de la modelización (Chuvieco, 2006) se ha realizado con cuatro parcelas de muestreo, de $8,3 \mathrm{~km}^{2}$ cada una, emplazadas aleatoriamente pero de manera no contigua. La validación ha demostrado una coincidencia en el 88,7\% de los casos, señalando errores por comisión en un $6 \%$ de las unidades.

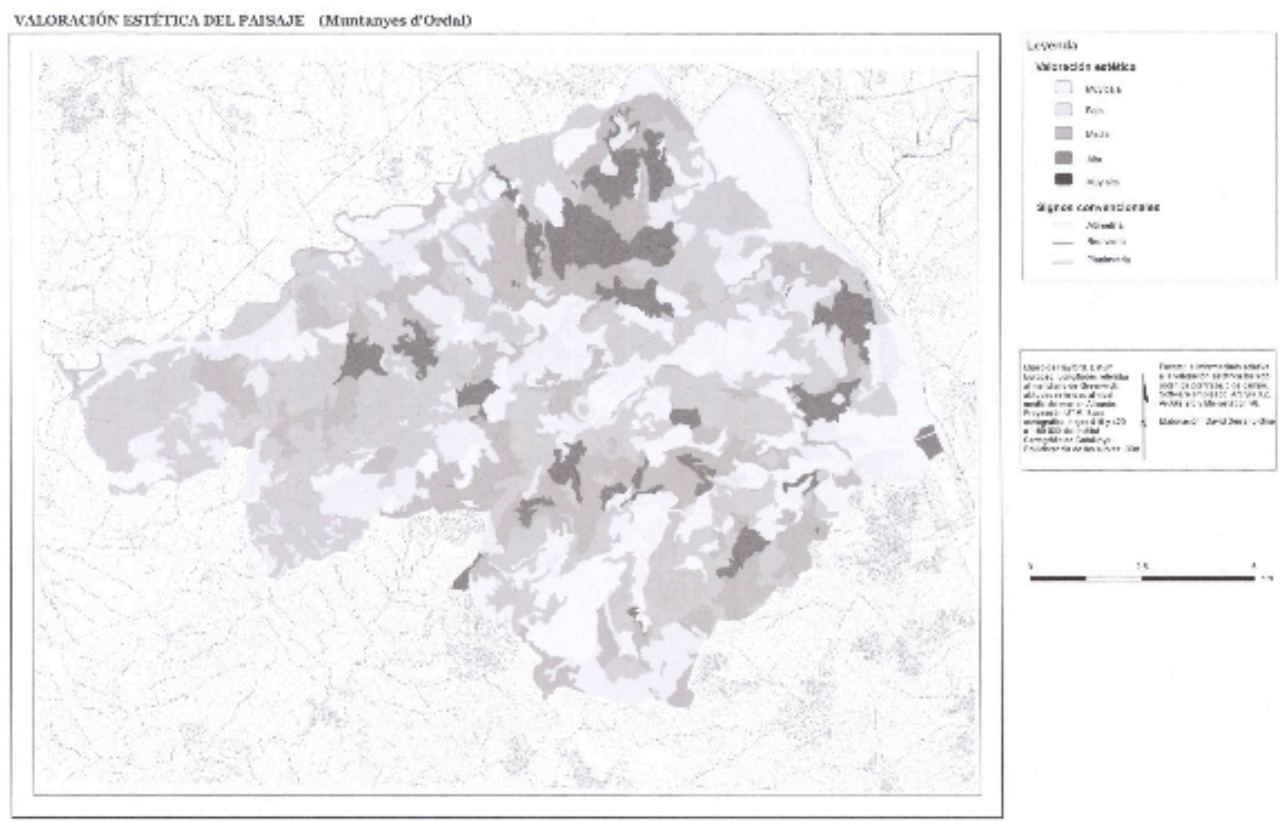

Figura 3. Valoración estética del paisaje (Muntanyes d’Ordal). 


\section{Consideraciones finales}

La valoración estética es un capítulo importante de cualquier estudio de paisaje, especialmente si se atiende a la importancia de estos trabajos en estudios aplicados y a la hora de valorar la calidad de vida de personas y sociedades. La diversidad de métodos existentes, la subjetividad inherente a toda valoración y la dificultad de establecer criterios universales merman la generalización de estos ejercicios. En cualquier caso, hay que tener presente la inexistencia de una metodología universal que excluya a otras, así como la necesidad de trabajar con métodos subjetivos pero sistemáticos.

En el área de estudio la modelización realizada indica, desde la consideración de los criterios y los parámetros escogidos, una abundancia de las unidades de valor estético medio, seguidas de las de valor alto. Las unidades con valoración negativa representan casi un 40\% de la totalidad, mientras que las de valoración muy alta con esfuerzo superan el 9\%. Ello se debe a la abundancia de elementos con ponderación baja, principalmente urbanizaciones de carácter extensivo, pero también masas forestales degradadas o poco evolucionadas.

En último lugar conviene señalar el elevado grado de aplicabilidad de este tipo de ejercicios, especialmente en lo que corresponde a gestión y ordenación del territorio y también a zonificación ambiental.

\section{Agradecimientos}

Durante el redactado de este artículo se ha contado con el apoyo institucional del Servei de Paisatge de la Universidad de Barcelona.

\section{Bibliografía}

AA.VV. (2004) Guía para la elaboración de estudios del medio físico. Madrid, Ministerio de Medio Ambiente.

Arriaza, M., Cañas Ortega, J.F., Cañas Madueño, J.A. y Ruiz-Avilés, P. (2004) Assessing the visual quality of rural landscapes. Landscape and Urban Planning, 69, $115-125$.

Bolòs, M. de (1992) Manual de ciencia del paisaje. Barcelona, Masson.

Cañellas, S., Waldrón, T. y Strand, R. (2006) La gestió pública del paisatge a Catalunya. En Cuadros, I. (dir) Paisatge, territori i socie- tat a les terres de parla catalana. Actes el V Congrés de la CCEP, Maó, CCEPC, p. 229241.

Cendrero, A. (1986) Mapa geocientífico de la provincia de Valencia. Valencia, Diputación de Valencia.

Fuente G. de la, Atauri, J.A. y Lucio, J.V. de (2006) Relationship between landscape visual attributes and spatial pattern indices: a test study in Mediterranean-climate landscapes. Landscape and Urban Planning, 77, 393-407.

García Asensio, J.M. y Cañas Guerrero, I. 
(2001) La valoración del paisaje. En Ayuga, F. (dir) Gestión sostenible de paisajes rurales. Madrid, Fundación Alfonso Martín Escudero, p. 33-51.

Gómez Limón, J. y Fernández, J.V. (1999) Changes in use and landscape preferentes on the agricultural-livestock landscapes of the central Iberian Peninsula (Madrid, Spain). Landscape and Urban Planning, 44, 165-174.

Mata, R., Gómez, J. y Fernández, S. (2001) El paisaje, calidad de vida y territorio Análisis Local, 37, 27-40.

González Bernáldez, F. (1981) Ecología y paisaje. Madrid, Blume.

González Bernáldez, F. (1993) La percepción de la calidad del paisaje. En EQUIP, Conferencias del I Congreso de Ciencia del paisaje. Barcelona, Universitat de Barcelona, p. 7-31.

INGEMISA (1987) Mapa geocientífico de la provincia de Alicante. València, Agència del Medi Ambient.

INGEMISA (1989) Mapa geocientífico de la provincia de Castellón. València, Agència del Medi Ambient.

Mallarach, J.M. (1999) Criteris i mètodes d'avaluació del patrimoni natural. Barcelona, Generalitat de Catalunya.

Martínez Vega, J., Martín, P. y Romero, R. (2003) Valoración del paisaje en la zona de especial protección de aves Carrizales y sotos de Aranjuez (Comunidad de Madrid). GeoFocus, 3, 1-21.

Nogué, J. (1986) Una lectura geogràficobumanista del paisatge de la Garrotxa. Girona, Diputació de Girona.

Ode, A., Tveit, M y Fry, G. (2008) Capturing landscape visual character using indicators: touching base with landscape aesthetic theory. Landscape Research, 33 (1), 89-117.

Paül, V. y Serrano, D. (2005) Muntanyes d'Ordal. El nom que no surt als mapes. Barcelona, Publicacion de l'Abadia de Montserrat.
Purcell, A.T. (1992) Abstract and specific physical attributes and the experience of landscape. Journal of Environmental Landscape, 34, 159-177.

Rochefort, R. (1974) La perception des paysages. L'Espace Géographique, 3, 637-648.

Scott, A. (2002) Assessing public perception of landscape: the LANDMAP experience. Landscape Research, 27 (3), 271-295.

Sánchez, J. (1995) Cartografía del potencial del medio natural de Gran Canaria. Las Palmas de Gran Canaria, Cabildo Insular de Gran Canaria-Universitat de ValènciaUniversidad de Las Palmas de Gran Canaria.

Serrano, D. y Piquer, M. (2006) Les Muntanyes d'Ordal i els moviments socials en defensa dels seus valors paisatgístics. $4^{e s}$ Jornades d'Estudi del Patrimoni del Baix Llobregat. Barcelona, Consell Comarcal del Baix Llobregat, p. 103-112.

Tarroja, A. (2006) Transformaciones territoriales y valoración social del paisaje. En Mata, R. (dir) El paisaje y la gestión del territorio. Barcelona, Diputació de Barcelona, p. 41-50.

Vaccaro, I. y Norman, K. (2008) Social sciences and landscape analysis: opportunities for the improvement of conservation policy design. Journal of Environmental Management, 88, 360-371.

Zoido, F. y Posocco, F. (1998) Política de paisaje: protección, ordenación y gestión. En Arias, J. y Fourneau, F. (eds) El paisaje mediterráneo. Le paysage méditerranéen. Il paesaggio mediterraneo. Granada, Universidad de Granada-Junta de Andalucía, p. 155-166

Zoido, F. (2002) El paisaje y su utilidad para la ordenación del territorio. En Zoido, F.; y Venegas, C. (eds) Paisaje y ordenación del territorio. Sevilla, Junta de AndalucíaFundación Duques de Soria, p. 21-32.

Zube, E. (1984) Environmental evaluation perception and public policy. Cambridge, Cambridge University Press. 